\title{
EFICACIA DEL USO DE PROBIÓTICOS EN EL TRATAMIENTO DE LA DIARREA ASOCIADA A ANTIBIOTERAPIA EN EL PACIENTE CRÍTICO.
}

\section{ENSAYO CONTROLADO Y ALEATORIZADO}

\author{
Irene Hidalgo Bravo \\ Ascensión Martínez López \\ Francisco José Martínez Rojo \\ Inmaculada Puerta Jiménez \\ María Carmen Pujante Rodríguez \\ María José Sánchez García
}





\section{EFICACIA DEL USO DE PROBIÓTICOS EN EL}

\section{TRATAMIENTO DE LA DIARREA ASOCIADA}

A ANTIBIOTERAPIA EN EL PACIENTE CRÍTICO.

ENSAYO CONTROLADO Y ALEATORIZADO

Autores:

Irene Hidalgo Bravo

Ascensión Martínez López

Francisco José Martínez Rojo

Inmaculada Puerta Jiménez

María Carmen Pujante Rodríguez

María José Sánchez García 


\section{${ }^{3}$ ciencias}

Editorial Área de Innovación y Desarrollo,S.L.

Quedan todos los derechos reservados. Esta publicación no puede ser reproducida, distribuida, comunicada públicamente o utilizada, total o parcialmente, sin previa autorización.

(C) del texto: Los autores

ÁREA DE INNOVACIÓN Y DESARROLLO, S.L.

C/ Els Alzamora, 17 - 03802 - ALCOY (ALICANTE) info@3ciencias.com

Primera edición: 1 de octubre de 2017

ISBN: 978-84-947995-0-1

DOI: http://dx.doi.org/10.17993/Med.2017.49 


\section{Índice}

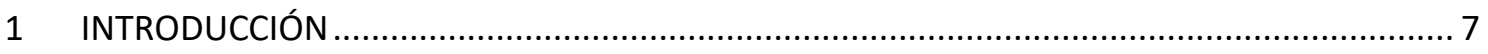

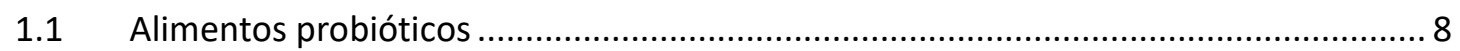

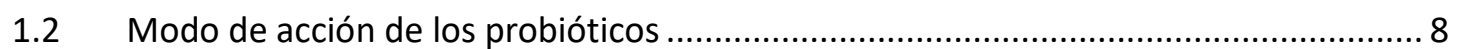

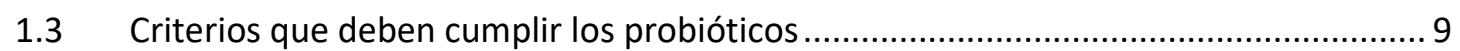

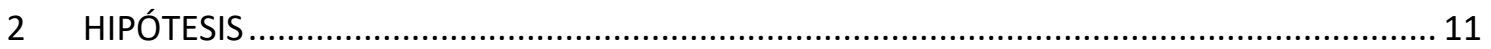

3 OBJETIVOS



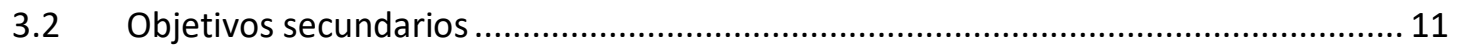

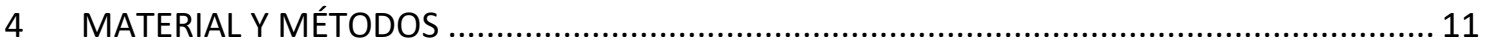

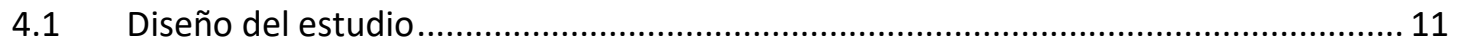

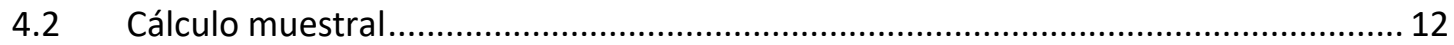

4.3 Criterios de inclusión y de exclusión .................................................................... 12

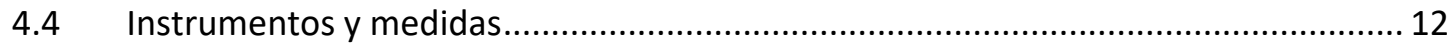

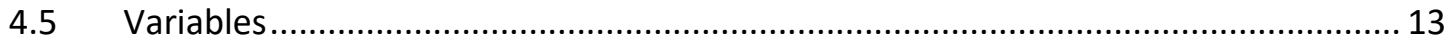

4.6 Procedimiento de recogida de datos ................................................................... 13

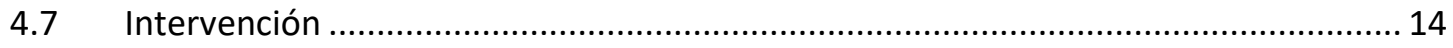

4.8 Plan de trabajo y calendario de actuación .................................................................. 14

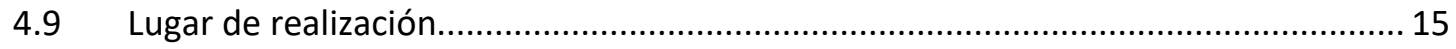

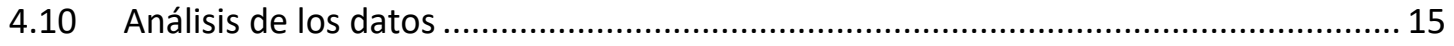

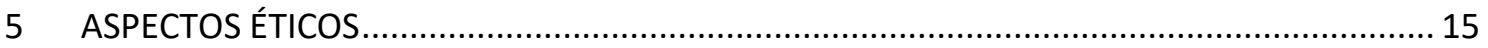



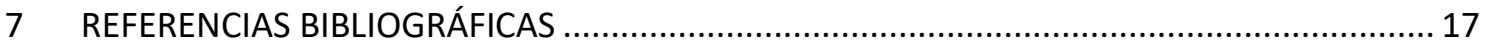

Anexo I. Hoja de información en el estudio del papel preventivo de la ingesta de antioxidantes sobre la enfermedad de Alzheimer 21

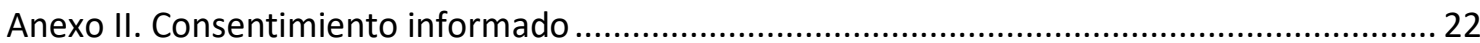





\section{INTRODUCCIÓN}

El tracto gastrointestinal (GI) constituye uno de los mayores sitios de exposición al medio ambiente exterior. Por lo tanto, el papel de la barrera de barrera es crucial (1). La barrera intestinal es una unidad funcional organizada como un sistema multicapa. Se divide en dos partes principales: una barrera física y una barrera funcional interna que diferencia entre patógenos y microorganismos comensales (2). Un componente de la barrera intestinal es la microbiota residente, mientras que la barrera subsiguiente es la capa de moco (3). La parte interna de la barrera intestinal está representada por una compleja red de células inmunes conocida como tejido linfoide asociado al intestino. Estas estructuras son esenciales para la actividad general de la barrera intestinal. Su deterioro permite el paso del contenido luminal en los tejidos subyacentes y por lo tanto en el torrente sanguíneo (intestino con fugas), dando como resultado la activación de la respuesta inmune y la inflamación. Esta alteración de la permeabilidad es la base de la patogénesis de muchas enfermedades gastrointestinales (1).

La diarrea es la deposición de cantidades aumentadas de heces blandas o líquidas. Las heces normales suelen ser sólidas debido a que el intestino delgado y el colon son altamente efectivos en la absorción de nutrientes, fluidos y sales diluidas, que son captados en el intestino superior. La diarrea ocurre cuando estos procesos se deterioran. Puede ser aguda (corto plazo, menos de 7 días), prolongada (una forma de diarrea aguda de más de 7 días) y crónica (a largo plazo) si los síntomas persisten más de 2 a 4 semanas, dependiendo de la edad. La diarrea también se clasifica como leve, moderada y severa sobre la base de la gravedad de los síntomas y la deshidratación producida (4).

En la Unidad de Cuidados Intensivos (UCI) son frecuentes los episodios de diarrea relacionados con una serie de infecciones (por ejemplo, Clostridium difficile o norovirus), ingesta de productos farmacéuticos (por ejemplo, laxantes y enemas) u otras causas no infecciosas (por ejemplo, insuficiencia pancreática y alimentación enteral continua) (5). La diarrea tiene un importante impacto sobre la dignidad del paciente, aumenta la carga de trabajo de enfermería y los costes de la $\mathrm{UCl}$, exacerbando la morbilidad del paciente a través de lesiones dérmicas, alteración de la absorción enteral y subsiguiente desequilibrio de líquidos y electrolitos. La diarrea representa una lesión gastrointestinal de grado II (IV) según las recientes directrices de la Sociedad Europea de Medicina Intensiva (ESICM), lo que indica que la digestión y la absorción son inadecuadas para satisfacer los requerimientos sistémicos de nutrientes y fluidos (6). 


\subsection{Alimentos probióticos}

El término "probióticos" puede ser definido como "microorganismos que, consumidos en una cantidad suficiente, producen una serie de beneficios en la salud del anfitrión" (7). Los microorganismos vivos con propiedades probióticas son bacterias que se encuentran disponibles en determinados alimentos, aunque también están disponibles comercialmente como suplementos probióticos $(8,9)$. Los probióticos más utilizados son los Lactobacillus, Bifidobacterias y las levaduras (ver tabla 1).

Tabla 1. Principales microorganismos probióticos.

\begin{tabular}{|c|c|c|c|}
\hline \multicolumn{3}{|c|}{ Bacterias del ácido láctico } & \multirow[t]{2}{*}{ Levaduras y otras } \\
\hline Lactobacillus & Bifidobacterias & Otras & \\
\hline L. acidophilus & B. adolescentis & $\begin{array}{l}\text { Enterococcus faecalis } \\
\text { Enterococcus }\end{array}$ & $\begin{array}{l}\text { Propionibacterium } \\
\text { freudenreichii }\end{array}$ \\
\hline L. bulgaricus & B. bifidus & $\begin{array}{l}\text { faecium } \\
\text { Sporolactobacillus }\end{array}$ & Bacillus cereus \\
\hline L. casei & B. breves & inulinus & Saccharomyces \\
\hline L. fermentum & B. infentis & & Boulardii \\
\hline L. helveticus & B. lactis & & \\
\hline L. plantarum & B. longum & & \\
\hline L. reuteri & & & \\
\hline L. rhamnosus & & & \\
\hline L. salivarus & & & \\
\hline L. lactis & & & \\
\hline L. crispatus & & & \\
\hline
\end{tabular}

Fuente: elaboración propia.

Entre las propiedades que tienen los microorganismos vivos para su uso como probióticos en seres humanos se incluye el ser similares a la microflora. Por este motivo, deben tener la capacidad de adherirse a las superficies de las células intestinales y de las glicoproteínas de las mucosas intestinales (mucina). El cultivo o cepa debe ser ácido, tolerante a la bilis y seguro para su uso clínico y alimentario $(10,11)$. La capacidad del probiótico para acumular los metabolitos microbianos también es un parámetro importante para la selección de la cepa adecuada (12).

Los probióticos tienen beneficios para la salud reconocidos y clínicamente validados (13) y son beneficiosamente eficaces contra una amplia gama de trastornos gastrointestinales (14).

\subsection{Modo de acción de los probióticos}

Efectos metabólicos de los probióticos. Los probióticos (microorganismos beneficiosos) desempeñan un papel central en la mejora de la salud del huésped (15), influyendo en su fisiología. Hay una serie de trastornos metabólicos que pueden ser tratados mediante varios 
probióticos con un modo de acción específico relacionado con el trastorno. Las cepas probióticas tienen la capacidad de disminuir el nivel de colesterol en sangre, mejorar la función de barrera del epitelio intestinal (16), disminuir las reacciones mutagénicas, disminuir la intolerancia a la lactosa, y disminuir el riesgo de cáncer (a través de la producción de peróxido de hidrógeno) (17).

Inmunomodulación. Las cepas probióticas (especialmente Lactobacillus GG) tienen varios efectos beneficiosos sobre las células secretoras de inmunoglobulina de sistema inmunitario (18). La administración de Lactobacillus tiene como resultado el fortalecimiento del sistema inmune, la modulación de la respuesta inmune de la mucosa, la mejora en la respuesta de las células secretoras de anticuerpos (19), la creación de equilibrio en el sistema inmune mediante la reducción de la severidad de los agentes patógenos (20), el incremento de los fagocitos (21), y también un control sobre el síndrome del intestino irritable y de los trastornos inflamatorios del intestino en general (22). No obstante, la estimulación de la respuesta inmune se considera como la function específica de los probióticos (23).

Normalización de la composición microbiana intestinal. Se sabe que en el momento del nacimiento, el tracto gastrointestinal del bebé es estéril, pero con el paso del tiempo la microbiota intestinal se desarrolla de forma estable hasta llegar a su estado funcional (24). Esta composición microbiana normal previene la unión de bacterias patógenas tales como Salmonella entérica, Escherichia coli, y el serotipo Typhimurium a las células epiteliales (25). La unión de microbios patógenos a las células epiteliales puede causar diarrea y otros trastornos gastrointestinales. Estas cepas también ayudan en la colonización de otras bacterias intestinales beneficiosas en lugar de la de patógenos (26).

Producción de los agentes antimicrobianos. Las cepas probióticas (especialmente Lactobacillus y las especies Lactococcus) inhiben el crecimiento de microorganismos patógenos (27) mediante la ocupación de la capa de la mucosa y la superficie de las células epiteliales (28), así como por la producción de agentes antimicrobianos tales como bacteriocinas o moléculas similares a bacteriocina, ácidos orgánicos, y el hidrógeno peróxido. La producción de estos agentes antimicrobianos suprime el crecimiento de microorganismos dañinos (17).

\subsection{Criterios que deben cumplir los probióticos}

Los probióticos, para ser considerados como tales, han de cumplir una serie de condiciones (29):

- Estar perfectamente caracterizado a nivel de género, especie y cepa y depositado en una colección de cultivo internacional y reconocida. 
- Ser seguro para el hospedador, determinado mediante ensayos in vitro y/o en animales de experimentación si es necesario.

- Ser viables en el momento de su consumo, aunque se ha reconocido que las células no viables pueden mediar algunos efectos beneficiosos a nivel fisiológico.

- Ser capaz de sobrevivir a su paso por el tracto gastrointestinal, determinándolo mediante una serie de pruebas estándar:

- Resistencia al pH gástrico y a sales biliares.

- Capacidad de adhesión a la mucosa y/o células epiteliales humanas o a líneas celulares.

- Actividad antimicrobiana frente a potenciales microorganismos patógenos.

- Deben proporcionar algún beneficio fisiológico demostrado mediante estudios llevados a cabo en los organismos diana.

Los probióticos pueden ser hongos, bacterias y levaduras, pero la gran mayoría son bacterias. Las cepas con propiedades benéficas a la salud humana y fuentes potenciales de probióticos pertenecen frecuentemente al género de Lactobacillus, Streptococcus y Bifidobacterium.

Estos microorganismos se implantan en alimentos, sobre todo en productos lácteos fermentados; convirtiéndose en una opción natural para mantener la flora intestinal en buen estado. Estos microorganismos vivos, aunque están incorporados especialmente en productos lácteos, también se pueden encontrar en otro tipo de alimentos fermentados como, por ejemplo, avena, verduras, embutidos o té (30).

La Consulta de Expertos FAO/OMS (31) sobre "Evaluación de las Propiedades Saludables y Nutricionales de los Probióticos en los Alimentos" propuso que se utilizaran las siguientes directrices, con respecto a las propiedades que deberían cumplir lo probióticos:

- Los microorganismos probióticos utilizados en los alimentos deberían ser capaces no sólo de sobrevivir al paso por el aparato digestivo, sino también de proliferar en el intestino. Esto significa que deberían ser resistentes a los jugos gástricos y poder crecer en presencia de bilis, en las condiciones existentes en los intestinos, o ser consumidos en un el estómago y a la exposición a la bilis.

- Los probióticos deben poder ejercer sus efectos beneficiosos en el huésped mediante su crecimiento y/o actividad en el cuerpo humano. 
- Es necesario perfeccionar los ensayos in vitro para comprobar y así garantizar la capacidad de los probióticos de funcionar en los seres humanos o bien realizar ensayos clínicos en humanos que permitan estudiar directamente dicha relación.

La dosis necesaria para los probióticos varía mucho dependiendo de la cepa y el producto. Aunque muchos productos ofrecen en el intervalo de 1-10 mil millones de UFC/dosis, algunos productos han demostrado ser eficaces a niveles más bajos, mientras que algunos requieren sustancialmente más. No es posible afirmar una dosis general de que se necesita para probióticos; la dosis tiene que ser sobre la base de estudios en humanos que muestran un beneficio para la salud (32).

\section{HIPÓTESIS}

El uso de probióticos en el paciente crítico con diarrea puede ser beneficioso ya que puede disminuir la sintomatología, reduciendo el número de días sin deposiciones diarreicas.

\section{OBJETIVOS}

\subsection{Objetivo primario}

Disminuir la duración del cuadro diarreico, definido como un menor número de días con diarrea, con el uso de probióticos en el paciente crítico.

\section{$3.2 \quad$ Objetivos secundarios}

1. Disminución de estancia del paciente crítico en $\mathrm{UCI}$.

2. Disminución en la aparición y gravedad de úlceras por decúbito.

3. Disminución de la infección nosocomial.

\section{MATERIAL Y MÉTODOS}

\subsection{Diseño del estudio}

Se realizará un ensayo prospectivo, controlado, aleatorizado y abierto, de dos grupos de pacientes palalelos. La asignación de cada paciente a uno de los grupos de estudio se realizará por medio de aleatorización simple. El proceso de aleatorización se realizará mediante generación de números pseudoaleatorios utilizando un programa informático. La ocultación de la secuencia de aleatorización se llevará a cabo mediante sobre sellado opaco. Uno de los grupos recibirá una intervención con probióticos para disminuir la duración del cuadro diarreico, mientras que el otro seguirá su dieta habitual. La duración del estudio será de 18 meses y se llevará a cabo en la UCI del Hospital Universitario Morales Meseguer. 


\subsection{Cálculo muestral}

Aceptando un riesgo alfa de 0,05 y un riesgo beta de 0,2 en un contraste bilateral, se precisan 63 sujetos en el primer grupo y 63 en el segundo para detectar una diferencia igual o superior a 1 unidad. Se asume que la desviación estándar en los días con diarrea es de 2. Se ha estimado una tasa de pérdidas de seguimiento del $0 \%$.

\subsection{Criterios de inclusión y de exclusión}

Los pacientes que formen parte del estudio deberán cumplir los criterios de inclusión y no los de exclusión.

\section{Criterios de Inclusión:}

a) Se incluirán todos los pacientes ingresados en UCI

b) Mayores de 18 años

c) En tratamiento antibiótico de amplio espectro durante al menos 48 horas previas a la inclusión en el estudio, que presenten cuadro diarreico, definido por la presencia de más de tres deposiciones y consistencia blanda-líquida, al día.

Criterios de exclusión. La presencia de al menos de uno de estos criterios supondrá la exclusión del paciente:

a) Paciente quirúrgico, intervenido de abdomen con necesidad de dieta absoluta.

b) Cualquier patología médica que condicione dieta absoluta.

c) Embarazo.

d) Situación crítica muy grave con posibilidad de muerte inmediata.

\subsection{Instrumentos y medidas}

Se aplicará un cuestionario de elaboración propia en el que se recogerán las siguientes medidas:

- Evaluación antropométrica, clínica, bioquímica y variables de resultados. Se medirán antes de la intervención los datos antropométricos de estatura $(m)$ y peso $(\mathrm{kg})$, calculando a continuación el índice de masa corporal (IMC, $\left.\mathrm{kg} / \mathrm{m}^{2}\right)$. La evaluación clínica incluirá la presión arterial sistólica y diastólica $(\mathrm{mg} / \mathrm{Hg})$. El análisis bioquímico se realizará mediante un análisis de sangre, extrayendo los valores de hemoglobina glicosilada (HbA1c) $(\mathrm{mmol} / \mathrm{mol})$, colesterol total (CT) $(\mathrm{mg} / \mathrm{dl})$, triglicéridos $(\mathrm{TG})(\mathrm{mg} / \mathrm{dl})$, lipoproteína de alta densidad (C-HDL) $(\mathrm{mg} / \mathrm{dl})$, y la lipoproteína de baja densidad (C-LDL) (mg/dl). Por último, se 
registrará la estancia hospitalaria en la UCI (días), el tiempo libre de diarrea (días), úlceras por presión (sí, no) y grado de las mismas (estadios I a IV). También se registrará la neumonía asociada a ventilación mecánica (sí, no), bacteriemia relacionada con catéter (sí, no) e infección del tracto urinario (sí, no).

- Datos sociodemográficos. Se administrará un cuestionario sociodemográfico que incluirá la edad (años) y el sexo (hombre, mujer). También se evaluarán los hábitos tóxicos como fumar (sí, no), alcohol (sí, no), así como la práctica de ejercicio físico (sí, no).

\subsection{Variables}

En la tabla 1 se encuentran una declaración de las variables del estudio y sus características.

Tabla 2. Variables del estudio.

\begin{tabular}{|c|c|c|c|}
\hline Variable & Descripción & Tipo & Valores/Unidades \\
\hline \multicolumn{4}{|c|}{ Datos sociodemográficos } \\
\hline Edad & Edad & Cuantitativa & Años \\
\hline Sexo & Sexo & Cualitativa & Hombre, mujer \\
\hline Fuma & Fuma & Cualitativa & sí, no \\
\hline Alcohol & Alcohol & Cualitativa & sí, no \\
\hline $\mathrm{EF}$ & Ejercicio físico & Cualitativa & sí, no \\
\hline \multicolumn{4}{|c|}{ Datos antropométricos, clínicos, bioquímicos y variables de resultados } \\
\hline Estatura & Estatura & Cuantitativa & $\mathrm{m}$ \\
\hline Peso & Peso & Cuantitativa & $\mathrm{kg}$ \\
\hline IMC & Índice de Masa Corporal & Cuantitativa & $\mathrm{kg} / \mathrm{m} 2$ \\
\hline $\mathrm{HbA1c}$ & Hemoglobina glicosilada & Cuantitativa & $\mathrm{mmol} / \mathrm{mol}$ \\
\hline CT & Colesterol total & Cuantitativa & $\mathrm{mg} / \mathrm{dl}$ \\
\hline TG & Triglicéridos & Cuantitativa & $\mathrm{mg} / \mathrm{dl}$ \\
\hline $\mathrm{C}-\mathrm{HDL}$ & $\begin{array}{l}\text { Lipoproteínas de alta } \\
\text { densidad }\end{array}$ & Cuantitativa & $\mathrm{mg} / \mathrm{dl}$ \\
\hline C-LDL & $\begin{array}{l}\text { Lipoproteínas de baja } \\
\text { densidad }\end{array}$ & Cuantitativa & $\mathrm{mg} / \mathrm{dl}$ \\
\hline Estancia & Días de estancia en UCl & Cuantitativa & Días \\
\hline Libre & Días libre de diarrea & Cuantitativa & Días \\
\hline UPP & Úlceras por presión & Cualitativa & sí, no \\
\hline Grado & Grado de UPP & Ordinal & Estadios I a IV \\
\hline Neumonía & Neumonía & Cualitativa & sí, no \\
\hline Bacteriemia & Bacteriemia & Cualitativa & sí, no \\
\hline Urinario & Infección tracto urinario & Cualitativa & sí, no \\
\hline
\end{tabular}

Fuente: elaboración propia. 


\subsection{Procedimiento de recogida de datos}

La recogida de datos y evaluaciones correspondientes se realizarán en la UCl del Hospital Universitario Morales Meseguer durante un período de 18 meses comprendido entre enero de 2018 y junio de 2019. Los participantes serán seleccionados tras el ingreso en esta unidad, donde se establecerá el cumplimiento de los criterios de inclusión y exclusión, solicitando, en su caso, la participación en el estudio. La evaluación será llevada a cabo por personal de enfermería que previamente habrá sido adecuadamente formado. La información de los cuestionarios será traspasada a una base de datos, realizándose una codificación para los datos personales que impidan la identificación del paciente por cualquier persona que no conozca dicho procedimiento, y que constará de un algoritmo basado en un código de centro, una fecha, la edad y el sexo. La persona responsable de la codificación de los datos personales garantizará la custodia y confidencialidad de los datos personales. Los datos del estudio serán volcados en una base inmediatamente después de cada recogida de datos para su posterior análisis estadístico.

\subsection{Intervención}

El probiótico administrado se compone de diferentes tipos de lactobacillus y se administrará a dosis de 2,5 g, dos veces al día vía oral o por sonda nasogástrica desde la tercera deposición diarreica en 24 horas hasta al menos 24 horas después de la disminución del número de deposiciones por debajo de tres al día. La administración se realizará vía oral o a través de la sonda nasogástrica.

Los pacientes del grupo control no recibirán tratamiento ni placebo alguno.

\subsection{Plan de trabajo y callendario de actuación}

Etapas de desarrollo y distribución de tareas. El estudio se realizará en tres etapas:

1. Diseño, preparación del equipo de investigación y de los medios materiales. Solicitud de permisos a las autoridades sanitarias correspondientes. Selección de los participantes.

2. Evaluación inicial, aplicación de la intervención. Registro de datos.

3. Análisis de los datos, elaboración de los informes de resultados y difusión de los mismos a través de publicaciones, congresos sobre efectos de ingesta de probióticos y otros ámbitos de difusión.

El estudio será coordinado por personal de enfermería, con la participación multidisciplinar de nutricionistas y gastroenterólogos, así como especialistas en análisis estadísticos, que 
participarán en las fases correspondientes del estudio. El personal sanitario pertenecerá a la UCl del Hospital Morales Meseguer.

\subsection{Lugar de realización}

El estudio será realizado en el Hospital Universitario Morales Meseguer, ubicado en la ciudad de Murcia (España), específicamente en la unidad de cuidados intensivos (UCI) de dicho hospital, que cuenta con 18 camas que atienden a pacientes médico-quirúrgicos.

\subsection{Análisis de los datos}

Las variables se expresan como medias \pm desviación estándar o mediana y cuartiles. Las variables cualitativas como frecuencia absoluta y relativa. La comparación entre variables se realiza mediante el test de T de Student y Chi-cuadrada de Pearson. Se calcula la diferencia de medias de días libres de diarrea entre ambos grupos con intervalos de confianza de $95 \%$. Se calculará el riesgo relativo para las variables cualitativas de resultados con sus intervalos de confianza al 95\%. Todos los contrastes se realizarán de forma bilateral y se considerara significativo un valor $p<0,05$. Se utilizará el paquete informático IBM-SPSS versión 22.0 para Windows.

\section{ASPECTOS ÉTICOS}

El estudio se presentará al Comité Éticos de Investigación del Hospital Universitario Morales Meseguer para su aprobación y conformidad.

La investigación se ajustará a los principios éticos de la Declaración de Helsinki, promulgada por la Asociación Médica Mundial que regula la investigación en la que se realiza experimentación con seres humanos. Los principios básicos son respeto por el individuo (artículo 8), así como el derecho a la autodeterminación y a tomar decisiones informadas (consentimiento informado) (artículos 20 a 22).

Los participantes serán informados de los objetivos y procedimientos mediante una hoja de información al paciente (ver Anexo I) y verbalmente, tras lo cual deberán firmar un consentimiento informado (Anexo II), asegurando la protección de los datos personales según lo establecido en la Ley Orgánica 15/1999, de 13 de diciembre, de Protección de Datos de Carácter Personal.

La investigación seguirá la guía de Buena Práctica Clínica, una norma internacional de calidad ética y científica aplicable al diseño, realización, registro y comunicación de los ensayos clínicos en los que participen seres humanos, promulgada por la Conferencia Internacional de 
Armonización. También se seguirán los principios de la Declaración de Helsinki en relación a la investigación Biomédica.

Con el fin de preservar la confidencialidad de la información sanitaria y personal de los participantes, la identidad de los mismos será codificada mediante un algoritmo de codificación que será custodiado por un miembro específicamente designado para este fin del equipo investigador. Ninguna otra persona tendrá acceso a dicha información.

\section{BENEFICIOS Y LIMITACIONES DEL ESTUDIO}

Los beneficios para los participantes y la población de estudio en general consisten en la mejora de la calidad de vida de los pacientes ingresados en las unidades de cuidados intensivos de los hospitales y que están en riesgo de padecer infección nosocomial con cuadros agudos de diarrea, mediante el uso de un tratamiento dietético de fácil aplicación y sin aparentes efectos secundarios, que además puede mejorar otros aspectos relacionados con la salud.

La principal limitación del estudio estriba en la escasez de participantes y en las dificultades para acceder a ellos, ya que se encuentran en un estado de salud por el que lo propios pacientes y sus familiares pueden poner objeciones a realizar este tipo de experimentación, a pesar de que no existe un riesgo para la salud de las personas ingresadas. 


\section{REFERENCIAS BIBLIOGRÁFICAS}

1. Scaldaferri F, Pizzoferrato M, Gerardi V, Lopetuso L, gasbarrini A. The gut barrier: new acquisitions and therapeutic approaches. J Clin Gastroenterol 2012;46:S12-7.

2. Lopetuso L, Scaldaferri F, Franceschi F, Gasbarrini A. The gastrointestinal microbiome - functional inferference between stomach and intestine. Best Pract Res Clin Gastroenterol 2014;28:995-1002.

3. Lozupone CA, Stombaugh JI, Gordon JI, Jansson JK, Knight R. Diversity, stability and resilience of the human gut microbiota. Nature 2012;489(7415):220-30.

4. Lopetuso L, Graziani C, Guarino A, Lamborghini A, Masi S, Stanghellini V. Gelatin tannate and tyndallized probiotics: a novel approach for treatment of diarrhea. Eur Rev Med Pharmacol Sci 2017;21:873-83.

5. Sabol VK, Carlson KK. Diarrhea: applying research to bedside practice. AACN Adv. Crit. Care 2007;18:32-44.

6. Reintam Blaser A, Malbrain MLNG, Starkopf J, Fruhwald S, Jakob SM, De Waele J, et al. Gastrointestinal function in intensive care patients: terminology, definitions and management. Recommendations of the ESICM Working Group on Abdominal Problems. Intensive Care Med 2012;38:384-94.

7. Mishra, S.K.; Mishra, P.; Saxene, M. Probiotics: An approach for better treatment. Res J Pharm Biol Chem Sci 2012, 3, 1042-61.

8. Leahy SC, Higgins DG, Fitzgerald GF, Van Sinderen D. Getting better with bifidobacteria. J Appl Microbiol 2005; 98:1303-15.

9. McNaught, C.E.; MacFie, J. Probiotics in clinical practice: A critical review of the evidence. Nutr Res 2001; 21:343-53.

10. Yan F, Polk DB. Probiotics and immune health. Curr Opin Gastroentrol 2011;27:496-501.

11. Eamonn M, Quigley M. Prebiotics and probiotics, modifying and mining the microbiota. Pharmacol Res 201061:213-8.

12. Gupta V, Garg R. Probiotics. Ind J Med Microbiol 2009; 27:202-9.

13. Bengmark S. Integrative medicine and human health, the role of pre, pro and synbiotics. Clin Transl Med 2012;1:6.

14. Yamano $T$, Lino $H$, Takada $M$, Blum S, Rochat F, Fukushima Y. Improvement of the human intestinal flora by ingestion of the probiotic strain Lactobacillus johnsonii La1. Br J Nutr 2006;95:303-12. 
15. Shafi A, Farooq U, Akram K, Jaskani M, Siddique F, Tanveer A. Antidiarrheal effect of food fermented by various strains of Lactobacillus. Compr Rev Food Sci Food Saf 2014;13:229-39.

16. Zyrek AA, Cichon C, Helms S, Enders C, Sonnenborn U, Schmidt MA. Molecular mechanisms underlying the probiotic effects of Escherichia coli Nissle 1917 involve ZO-2 and PKCZ redistribution resulting in tight junction and epithelial barrier repair. Cell. Microbiol 2007;9:804-16.

17. Ng SC, Hart AL, Kamm MA, Stagg AJ, Knight SC. Mechanisms of action of probiotics: Recent advances. Inflamm Bowel Dis 2009;15:300-10.

18. Nova E, Wärnberg J, Gómez-Martínez S, Díaz LE, Romeo J, Marcos A. Immunomodulatory effects of probiotics in different stages of life. $\mathrm{Br} J$ Nutr 2007;98:S90-5.

19. Rinne M, Kalliomaki M, Arvilommi H, Salminen S, Isolauri E. Effect of probiotics and breast feeding on the Bifidobacterium and Lactobacillus/Enterococcus microbiota and humoral immune responses. J Pediatr 2005;147:186-91.

20. Bengmark S, Gil A. Bioecological and nutritional control of disease: Prebiotics, probiotics and synbiotics. Nutr Hosp 2006;21:72-84.

21. Shu Q, Gill HS. Immune protection mediated by the probiotic Lactobacillus rhamnosus HNO01 (DR20) against Escherichia coli 0157: H7 infection in mice. FEMS Immunol Med Microbiol 2002;34:59-64.

22. Gill HS, Rutherfurd KJ, Cross ML, Gopal PK. Enhancement of immunity in the elderly by dietary supplementation with the probiotic Bifidobacterium lactis HN019. Am J Clin Nutr 2001;74:833-9.

23. Nissen L, Chingwaru W, Sgorbati B, Biavati B, Cencic A. Gut health promoting activity of new putative probiotic/protective Lactobacillus spp. strains: A functional study in the small intestinal cell model. Int J Food Microbiol 2009;135:288-94.

24. Fanaro S, Chierici R, Guerrini P, Vigi V. Intestinal microflora in early infancy: Composition and development. Acta Paediatr 2003;91:48-55.

25. Penders J, Thijs C, Vink C, Stelma FF, Snijders B, Kummeling I, et al. Factors influencing the composition of the intestinal microbiota in early infancy. Pediatrics 2006;118:511-21.

26. Turnbaugh PJ, Ley RE, Hamady M, Fraser-Liggett CM, Knight R, Gordon Jl. The human microbiome project. Nature 2007;449:804-10. 
27. Burr GS, Gatline DM, Ricke S. Microbial ecology of gastrointestinal tract and the potential application of probiotics and prebiotics in finfish aquaculture. J World Aqua Soc 2005;36:425-36.

28. Shiba T, Aiba Y, Ishikawa H, Ushiyama A, Takagi A, Mine T, Koga Y. The suppressive effect of bifidobacteria on Bacteroides vulgatus, a putative pathogenic microbe in inflammatory bowel disease. Microbiol Immunol 2003;47:371-8.

29. Saulnier D, Spinler J, Gibson G, Versalovic J. Mechanisms of probiosis and prebiosis: considerations for enhanced functional foods. Curr Opinion Biotechnol 2009;20(2):135-41.

30. Silveira Rodríguez MB, Morenereo Megías S, Molina Baena B. Alimentos Funcionales y Nutrición Óptima. ¿Cerca o Lejos? Rev Esp Salud Pública 2003;77(3):148-53.

31. Probióticos en los alimentos. Roma: Organización de las Naciones Unidas para la

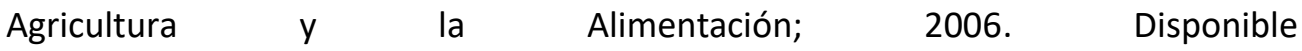
ftp://ftp.fao.org/docrep/fao/009/a0512s/a0512s00.pdf

32. Guarner F, Khan Aamir G, Garisch J. Guía práctica Organización Mundial de Gastroenterología: Probióticos y prebióticos. Milwaukee: Organización Mundial de Gastroenterología; 2011. 
Eficacia del uso de probióticos en el tratamiento de la diarrea asociada a antibioterapia en el paciente crítico. Ensayo controlado y aleatorizado 


\section{Anexo I. Hoja de información en el estudio del papel preventivo de la ingesta de antioxidantes sobre la enfermedad de Alzheimer}

El propósito de esta hoja de información es proporcionar a los participantes en esta investigación con una clara explicación de la naturaleza de la misma, así como de su rol en ella como participantes.

La meta de este estudio es conocer el posible efecto de la ingesta de probióticos en la duración de diarrea en pacientes hospitalizados en $\mathrm{UCI}$.

Se realizará un ensayo experimental prospectivo en personas ingresadas en $\mathrm{UCl}$, con dos grupos: el grupo experimental recibirá un suplemento de probióticos (lactobacillus). El grupo de control seguirá con la dieta habitual.

La intervención administrada no produce efectos adversos sobre la salud. En todo caso se realizará un amplio examen clínico y bioquímico con el fin de descartar la participación de aquellas personas no aptas para recibir los tratamientos.

Si usted accede a participar en este estudio, se le informa de que los datos recopilados serán tratados de forma anónima y confidencial, y que dicha información será usada únicamente con fines investigadores. La participación en este estudio es estrictamente voluntaria. La información que se recoja será confidencial y no se usará para ningún otro propósito fuera de los de esta investigación. Su participación será codificada usando un número de identificación y, por lo tanto, será anónima. Una vez analizado el caso, los archivos con las grabaciones se destruirán.

Si tiene alguna duda sobre este proyecto, puede hacer preguntas en cualquier momento durante su participación en él. Igualmente, puede retirarse del proyecto en cualquier momento sin que eso lo perjudique en ninguna forma. 
Anexo II. Consentimiento informado

CONSENTIMIENTO INFORMADO PARA LA REALIZACIÓN DE ENSAYOS CLÍNICOS, PROYECTOS DE INVESTIGACIÓN Y ESTUDIOS GENÉTICOS

(Versión y fecha del Cl)

TÍTULO DEL ESTUDIO:

CÓDIGO DEL PROMOTOR:

PROMOTOR:

INVESTIGADOR PRINCIPAL (Médico del estudio, si excepcionalmente se trata de otro profesional se debe indicar. Incluir nombre, servicio y teléfono):

CENTRO:

Yo, (nombre y apellidos),

$\square$ He leído la hoja de información que se me ha entregado.

$\square$ He podido hacer preguntas sobre el estudio.

$\square$ He recibido suficiente información sobre el estudio.

$\square$ He hablado con: (nombre del investigador).

$\square$ Comprendo que mi participación es voluntaria.

$\square$ Comprendo que puedo retirarme del estudio (si se recogen muestras, añadir:) y solicitar la destrucción de mi muestra, siempre y cuando no haya sido anonimizada:

Cuando quiera.

Sin tener que dar explicaciones.

Sin que esto repercuta en mis cuidados médicos.

$\square$ Comprendo que, si decido retirarme del estudio, los resultados obtenidos hasta ese momento podrán seguir siendo utilizados (si se recogen muestras, añadir:) pero que no se realizarán nuevos análisis de mi muestra, siempre y cuando no haya sido anonimizada.

En el caso de que los resultados de la investigación proporcionen datos que me puedan interesar a mí o a mis familiares: (indicar una de las casillas)

$\square$ Quiero ser informado.

$\square$ No quiero ser informado, pero acepto que mi médico contacte con mis familiares si dichos resultados les pueden afectar.

$\square$ Comprendo que tengo los derechos de acceso, rectificación, cancelación y oposición a mis datos de carácter personal de acuerdo con lo dispuesto en la Ley Orgánica 15/1999 de Protección de Datos de Carácter Personal. 
$\square$ Presto libremente mi conformidad para participar en el estudio y doy mi consentimiento para el acceso y utilización de mis datos en las condiciones detalladas en la hoja de información al paciente.

(En caso de solicitar muestras para el proyecto, y tener previsto almacenarlas para otros estudios, deberá incluir el siguiente párrafo, ofreciendo al participante una o varias de las siguientes opciones, de acuerdo con la información facilitada en la hoja de información)

Al término de la investigación mi muestra podrá ser:

$\square$ Destruida

Anonimizada

$\square$ Incorporada en una colección cuyo responsable es el investigador (indicar nombre completo del investigador responsable de la colección), que se encuentra en (indicar lugar), para continuar ser utilizada en el estudio de (indicar la línea de investigación).

$\square$ Almacenada en el biobanco (indicar cuál) para poder ser utilizada en otras investigaciones, posiblemente no relacionadas con el estudio inicial para el cual consintió.

Firma del paciente: Firma del investigador:

Nombre: Nombre:

Fecha: Fecha: 
Eficacia del uso de probióticos en el tratamiento de la diarrea asociada a antibioterapia en el paciente crítico. Ensayo controlado y aleatorizado 

Medicina y Salud 